\title{
Automatic Detection of Secondary Creases in Fingerprints
}

\author{
David Vernon \\ Department of Computer Science \\ Trinity College Dublin \\ Ireland
}

\begin{abstract}
Human fingerprints comprise a series of whorls or ridges. In some special cases, these whorls are broken by so-called 'secondary creases': co-linear breaks across a sequence of adjacent ridges. A technique to automatically detect such creases in fingerprints is described. This technique utilizes a combination of spatial filtering and region-growing to identify the morphology of the locally fragmented fingerprint image. Regions are then thinned to form a skeletal model of the ridge structure. Creases are characterised by co-linear terminations on ridges and are isolated by analysing the Hough transform space derived from the ridge end points. Empirical results using both synthetic and real data are presented and discussed.
\end{abstract}

Keywords: Fingerprint analysis, secondary creases, Hough transform, virtual lines, Laplacian of Gaussian, spatial scale.

\section{Secondary Creases}

Human fingerprints comprise a series of whorls or ridges. The imprint of these ridges forms a quasi-contiguous locally-linear series of ink prints where the surface of the skin has come into contact with the paper on which the fingerprint is formed (see figure 1). For most people, these ridges are well-formed and unbroken. However, in some people, these ridges are broken by a crease where no skin has been inked and come into contact with the paper. These co-linear breaks in the whorls of the fingerprint are called secondary creases (see figure 2). It is a working hypothesis that the presence of such secondary creases form a physical marker for certain human disorders. This paper discusses the research and development of an automated technique to detect and isolate secondary creases so that this working hypothesis can be verified without relying on human interpretation of the morphology of the fingerprint. 


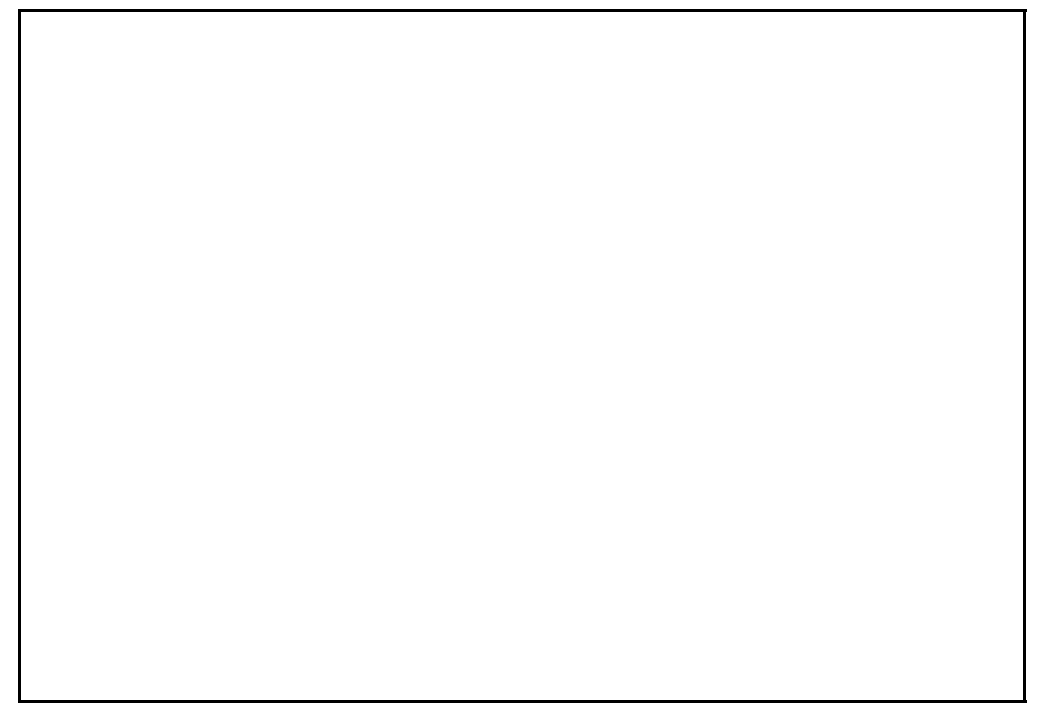

Figure 1: A normal human fingerprint

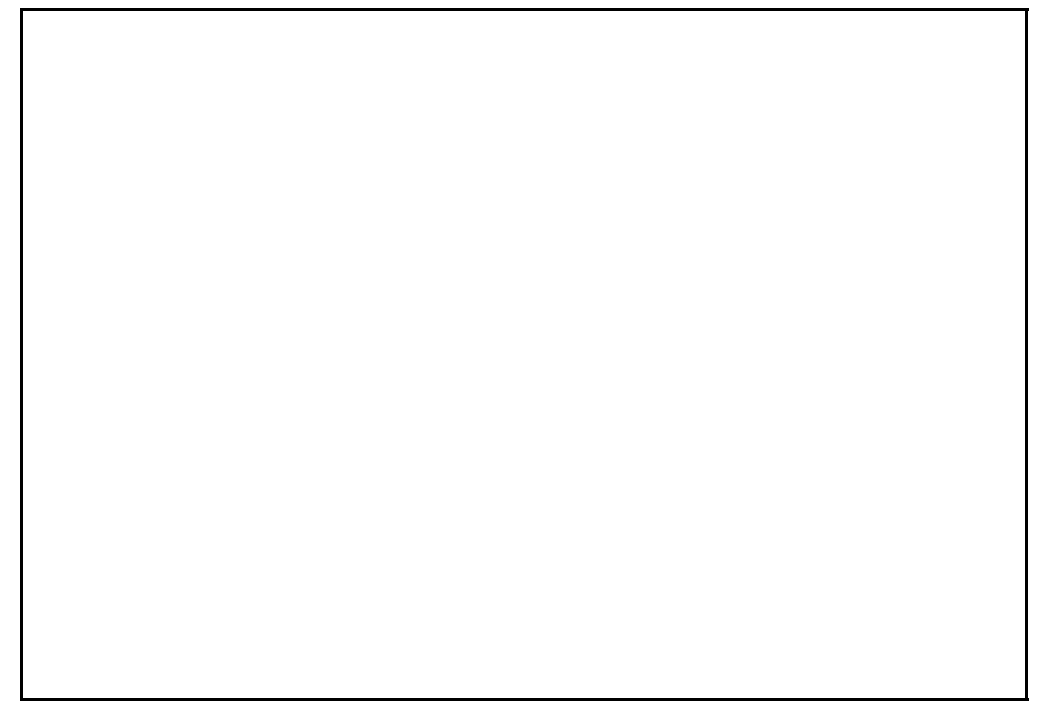

Figure 2: A human fingerprint with secondary creases 


\section{Segmentation}

Before any automatic analysis of the morphology of the fingerprint can be accomplished, it if first necessary to identify and label those parts of the image of the fingerprint which correspond to the ridges or whorls, i.e. the inked part of the paper.

In normal circumstances where the object of interest, i.e. the ridges, are of a distinctly different grey-level to the background, i.e. the paper on which the fingerprint is imprinted, this process of segmentation could effected by 'thresholding'. In this process, the grey-level of each pixel is compared with an appropriate reference level - the threshold - and the pixel is then assigned a value or 255 depending on whether it is greater or less than the threshold. The resultant binary image comprises pixels of grey-level 0 , signifying that a pixel represents a ridge, or 255, signifying that a pixel represents the paper. Unfortunately, this straightforward approach is not practicable for two reasons.

Firstly, the level of inking of the finger can vary considerably and consequently the 'blackness' of the print varies from fingerprint to fingerprint and from region to region in a given fingerprint. Secondly, a segmented image of a fingerprint which has been generated by thresholding is extremely fragmented in the sense that a single ridge is broken up into many (in the order of tens or hundreds) isolated, non-adjacent, blobs or regions. This is a natural consequence of the textured nature of the surface of the skin which forms the whorls. While the problem of inhomogeneous inking can be solved through the use of dynamic thresholding [1] wherein the threshold is a function of the image coordinates, the second difficulty of ridge fragmentation is more problematic. An informal investigation of the feasibility of identifying the global structure of each whorl through accepted techniques, such as morphological opening [2], yielded no useful solution.

In the work described here, a robust, if computationally-expensive, solution is employed which addresses simultaneously the problems of fragmentation and segmentation. This technique utilizes a combination of spatial filtering and region-growing to identify the morphology of the fingerprint image.

Ridges, as entities in themselves, in the fingerprint are substantially larger than the fragments which make up the ridge; that is, they are well-represented by the lower spatial frequencies comprising the image. By attenuating the higher spatial frequencies, the fragmented image detail is removed and the global structure of the ridge morphology is retained. This can be best accomplished [3] by convolving the fingerprint image with a two-dimensional Gaussian function:

$$
I(x, y) * G(x, y)
$$

where $I(x, y)$ represents the image intensity at a point $(x, y)$ and $G(x, y)$ is the 2-D Gaussian function, of a given standard deviation $\sigma$, defined by:

$$
G(x, y)=\frac{1}{2 \pi \sigma^{2}} \exp ^{-\frac{\left(x^{2}+y^{2}\right)}{2 \sigma^{2}}}
$$


The value of $\sigma$ governs the spatial scales which are retained: the larger the value of $\sigma$, the larger the scale of the objects which are represented in the filtered image. In all of the results cited in this paper, $\sigma=7.0$ pixels. This value was determined empirically through calibration procedure, based on the normal distance between the ridges.

The ridges are isolated through the use of a Laplacian second-derivative edge detection filter

$$
\nabla^{2}=\frac{\partial^{2}}{\partial x^{2}}+\frac{\partial^{2}}{\partial y^{2}}
$$

i.e. the sum of second-order, unmixed, partial derivatives.

The evaluation of the Laplacian and the convolution with the Gaussian commute so that the segmentation and selection of spatial scale can be effected with a single filter: the Laplacian of Gaussian[4]:

$$
\nabla^{2}(I(x, y) * G(x, y))=\nabla^{2} G(x, y) * I(x, y)
$$

Furthermore, this 2-D convolution is separable into four 1-D convolutions:

$$
\nabla^{2}(I(x, y) * G(x, y))=G(x) *\left(I(x, y) * \frac{\partial^{2}}{\partial y^{2}} G(y)\right)+G(y) *\left(I(x, y) * \frac{\partial^{2}}{\partial x^{2}} G(x)\right)
$$

which facilitates computational savings in the application of the filter from on the order of $n^{2}$ operations to $4 n$ operations, where $n$ is the size of the filter kernel. Since $n$ can be quite large ( $n=49$ in the implementation described in this paper) such savings can be quite significant. The Laplacian of Gaussian operator yields thin continuous closed contours of zero-crossing points which bound regions in the filtered image. These regions are recursively nested, with each region having, alternately, an opposite sign (positive or negative) as one descends through the nesting (see [5]). In this work on the detection of secondary creases, segmentation is achieved by identifying each region by its sign, computing the area of each region, and isolating the region with the largest area. This is the background region and all other regions are deemed to be 'ridge regions'. This segmentation then is represented as a binary image; for example, see figure 3 (b) through 8 (b).

\section{Morphological processing: thinning and isola- tion of ridge termination points}

Once the elongated ridge regions have been isolated, it is necessary to identify locations of their ends. This is accomplished by morphological processing, thinning the region to form skeletons which are one pixel wide, and then by identifying the end-points of these skeletons. The procedure can be summarized as follows (see [6] for further details.) Let $X$ denote the set of points (pixels) which 


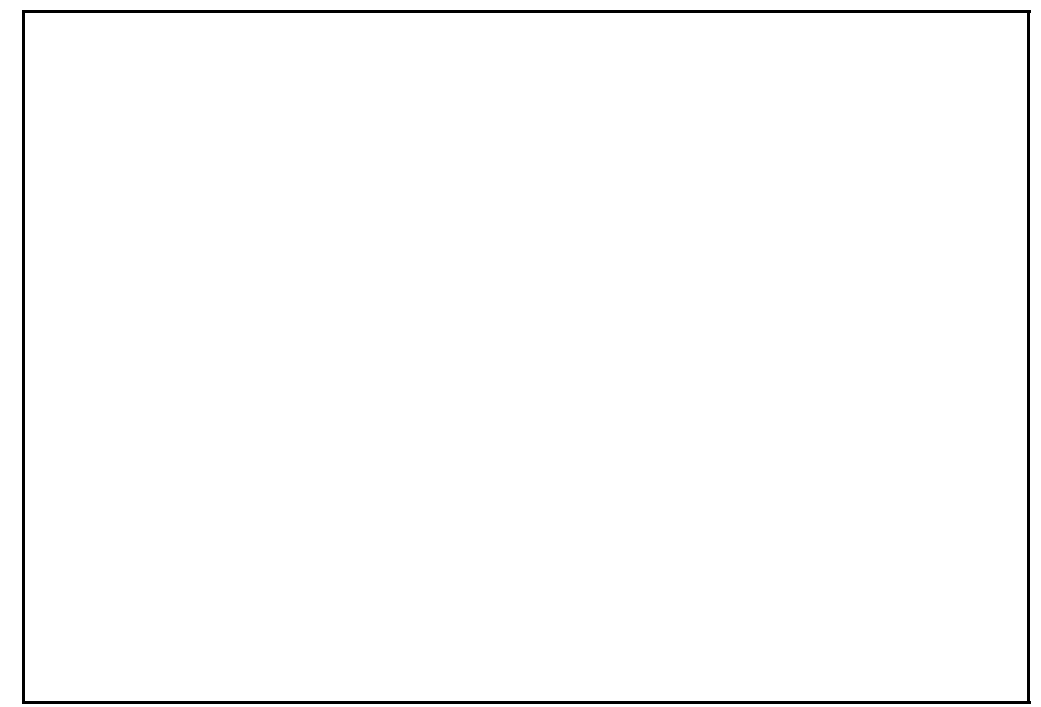

Figure 3: (a) Top left: grey-level image of a section for a fingerprint. (b) Top right: Ridge regions derived from Laplacian of Gaussian $\nabla^{2} G$ of image; $\sigma=7.0$ pixels. (c) Bottom left: Hough transform space derived from end-points of skeleton of ridge regions. (d) Bottom right: Crease line superimposed on the skeleton of ridge regions.

comprise the ridge regions. The thinning of this set $X$ is accomplished by the morphological filtering of the set $X$ with a sequence of structuring elements $L$, as follows.

$$
X \bigcirc\left\{L^{i}\right\}
$$

that is

$$
\left(\left(\left(\ldots\left(X \bigcirc L^{1}\right) \bigcirc L^{2}\right) \bigcirc L^{3}\right) \ldots \bigcirc L^{i}\right)
$$

where $X \bigcirc L$ is defined:

$$
X \bigcirc L=X \backslash X \otimes L
$$

The operator $\otimes$ denotes the hit or miss transformation. This transformation is defined

$$
X \otimes L=\left\{x \mid L_{x}^{f} \subset X ; L_{x}^{b} \subset X^{C}\right\}
$$

where $L_{x}^{f}$ is that subset of $L$, translated to point $x$, whose elements belong to the 'foreground' (ridge) and $L_{x}^{b}$ is the subset of $L$, translated to point $x$, whose elements belong to the 'background' (i.e. $L^{f} \cap L^{b}=\emptyset$ ). $X^{C}$ denotes the set complement of $X$. A point $x$ belongs to the hit or miss transformtion if and only if $L_{x}^{f}$ is included in $X$ and $L_{x}^{b}$ is included in the complement of $X$. Thus, $X \otimes L$ defines the points where the structuring element $L$ exactly matches (hits) the set $X$, i.e. the ridge pixels in the image. The set $X \bigcirc L$, then, is the set $X$ 


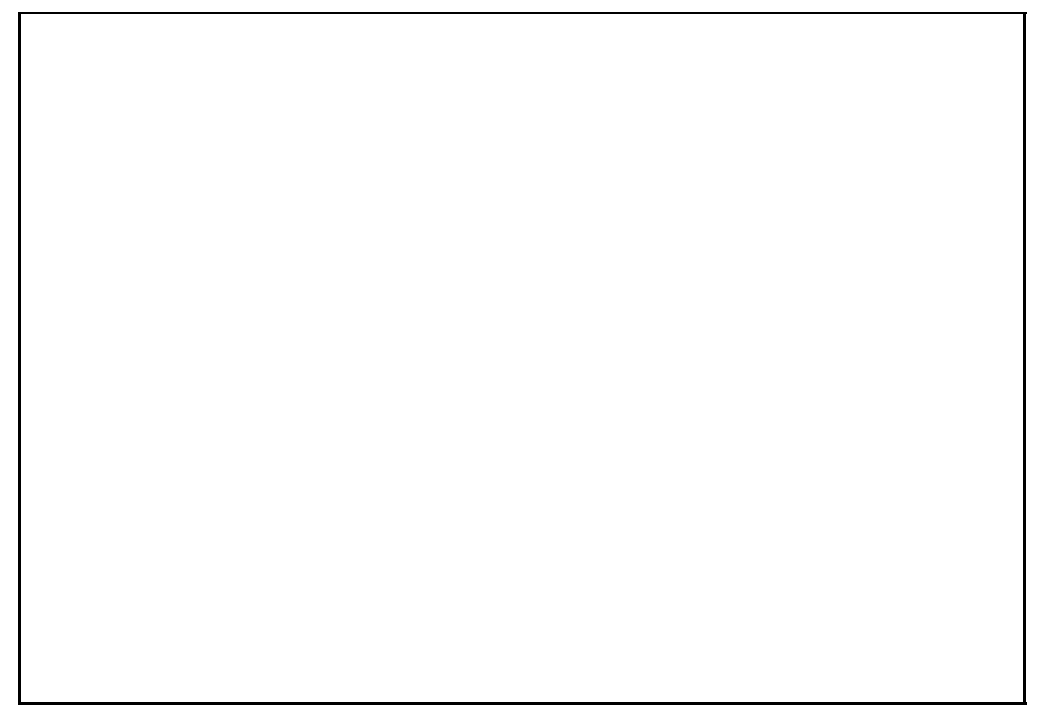

Figure 4: (a) Top left: grey-level image of a section for a fingerprint. (b) Top right: Ridge regions derived from Laplacian of Gaussian $\nabla^{2} G$ of image; $\sigma=7.0$ pixels. (c) Bottom left: Hough transform space derived from end-points of skeleton of ridge regions. (d) Bottom right: Crease line superimposed on the skeleton of ridge regions (note: $\delta_{r}^{2}=0.2$; see section 4.4 and compare also with figure 3 ). 


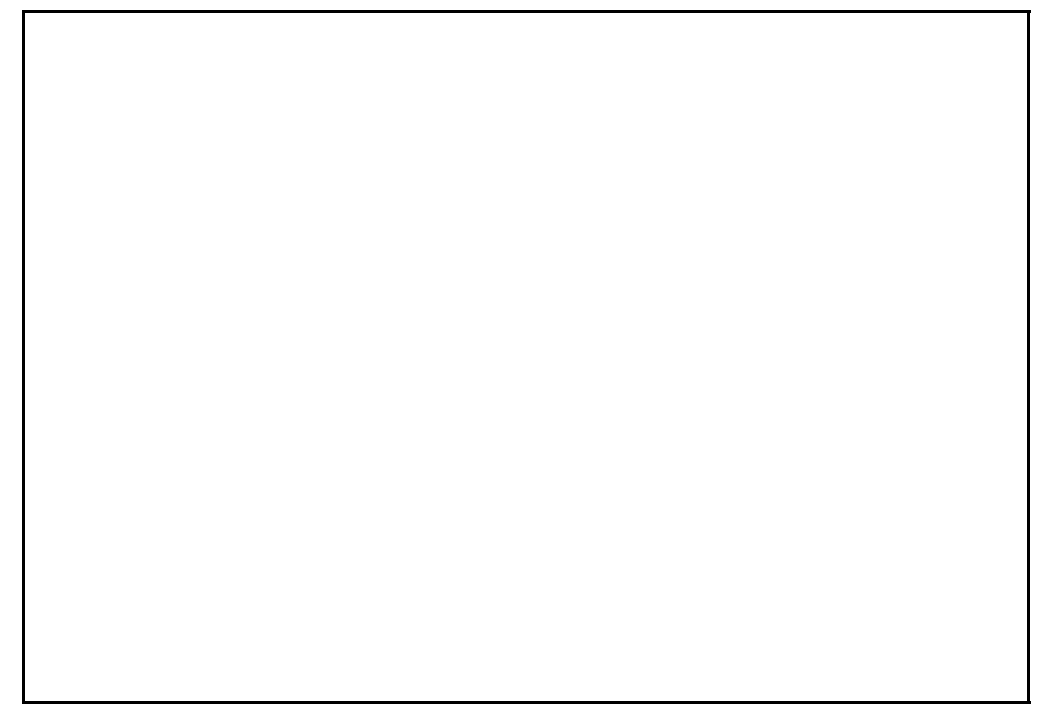

Figure 5: (a) Top left: grey-level image of a section for a fingerprint. (b) Top right: Ridge regions derived from Laplacian of Gaussian $\nabla^{2} G$ of image; $\sigma=7.0$ pixels. (c) Bottom left: Hough transform space derived from end-points of skeleton of ridge regions. (d) Bottom right: Crease line superimposed on the skeleton of ridge regions.

less the set of points in $X$ which hit $L$. Thus, if $X \otimes L$ identifies border points, and $L$ is appropriately structured to maintain the connectivity of a set, then repeated application of the thinning process successively removes border points from a set until the skeleton is achieved. At this point, further application of the thinning transform yields no change in the skeletal set. The sequence $\{L\}$ which is used for thinning is based on a single structuring element and is generated by rotating the structuring element (through $360^{\circ}$ in increments of $45^{\circ}$ ). This sequence $\{L\}$ is shown in figure 9 . The thinning algorithm then amounts to the repeated transformation of a set $X_{i} \rightarrow X_{i+1}$ defined:

$$
X_{i+1}=\left(\left(\left(\ldots\left(X_{i} \bigcirc L^{1}\right) \bigcirc L^{2}\right) \bigcirc L^{3}\right) \ldots \bigcirc L^{8}\right)
$$

The skeleton is achieved when $X_{i}=X_{i+1}$. Initially, $X_{0}=X$, i.e., the original (unthinned) segmented binary image. Examples of thinned ridges can be seen in figures 3 (d) through 8 (d).

Given a skeleton $X$, we then identify the end-points, i.e. points which are connected to just one other point, using the hit or miss transform and an appropriate set of structuring elements $\{E\}$, shown in figure 10 . Thus, the endpoints 


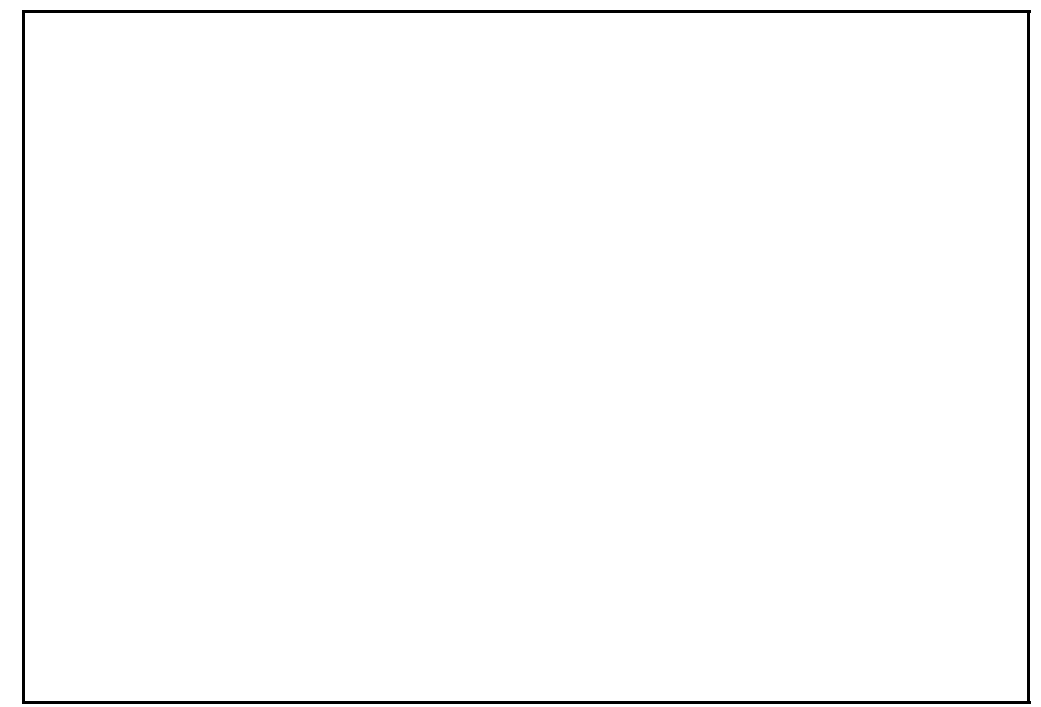

Figure 6: (a) Top left: grey-level image of a section for a fingerprint. (b) Top right: Ridge regions derived from Laplacian of Gaussian $\nabla^{2} G$ of image; $\sigma=7.0$ pixels. (c) Bottom left: Hough transform space derived from end-points of skeleton of ridge regions. (d) Bottom right: Crease line superimposed on the skeleton of ridge regions.

of the skeleton are given by:

$$
Y=\bigcup_{i=0}^{8} X \otimes E^{i}
$$

That is, the set of end-points is the union of all those points which hit with one of these endpoint structuring elements.

\section{Hough Transform}

\subsection{Computing the Hough Transform}

The technique for the detection of secondary creases described in this paper is based on the assumption that such creases are characterized by co-linear ridge ends. Having extracted the morphology of the ridges and having identified the locations of the end-points of the segmented ridges, it now remains to group these end-points according to a co-linearity criteria: in effect, to find the virtual line formed by the ridge ends. The Hough transform [7] is used to accomplish this. The computation of the Hough transform for the detection of lines is quite straight-forward although, as we shall see in the next section, post-processing 


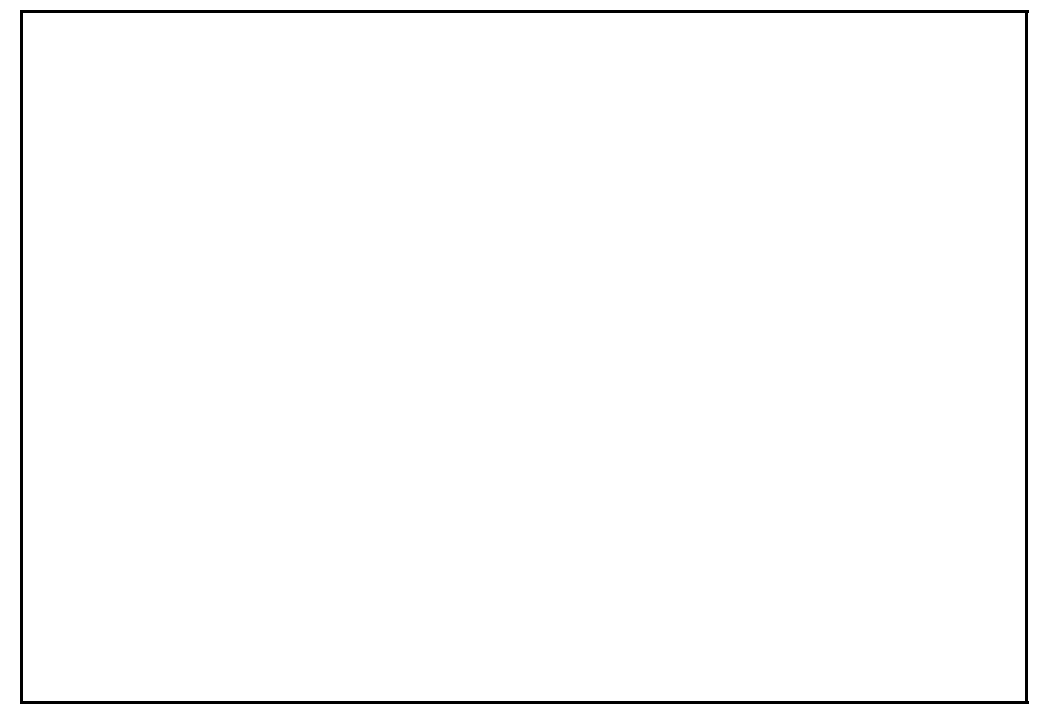

Figure 7: (a) Top left: grey-level image of a section for a fingerprint. (b) Top right: Ridge regions derived from Laplacian of Gaussian $\nabla^{2} G$ of image; $\sigma=7.0$ pixels. (c) Bottom left: Hough transform space derived from end-points of skeleton of ridge regions. (d) Bottom right: Crease line superimposed on the skeleton of ridge regions.

in the Hough transform space is required to effect reliable and robust extraction of lines.

The equation of a straight line is given in parametric form by the equation:

$$
x \cos \theta+y \sin \theta=r
$$

where $r$ is the length of a normal to the line from the origin and $\theta$ is the angle this normal makes with the $X$-axis. For a given line, $r$ and $\theta$ are known. In this case, however, $r$ and $\theta$ are unknown since we do not yet know which are the crease lines but we have several specific samples of $x$ and $y-x_{i}$ and $y_{i}$, say - which are given by the coordinates of the ridge end-points. In the Hough transform, the solution to equation 11 is computed for each $\left(x_{i}, y_{i}\right)$ pair, yielding a set of values for $r$ and $\theta$. These values are recorded by incrementing an element of a 2-D array, known as the Hough accumulator, for each $(r, \theta)$. From a computational point of view, this is done quite simply by computing the value of $\theta$ from equation 11 for all values of $r$, knowing $x_{i}$ and $y_{i}$. This solution-set is, in effect, a sinusoidal curve in the $r-\theta$ space, i.e., in the Hough transform space. The transform is computed for all ridge end-points $\left(x_{i}, y_{i}\right)$ and end-points which are co-linear will all have a single value of $r$ and $\theta$ in common; that is, the solution-set sinusoidal curves will intersect in a single point in the Hough transform space. Such points of intersection are characterized by local 


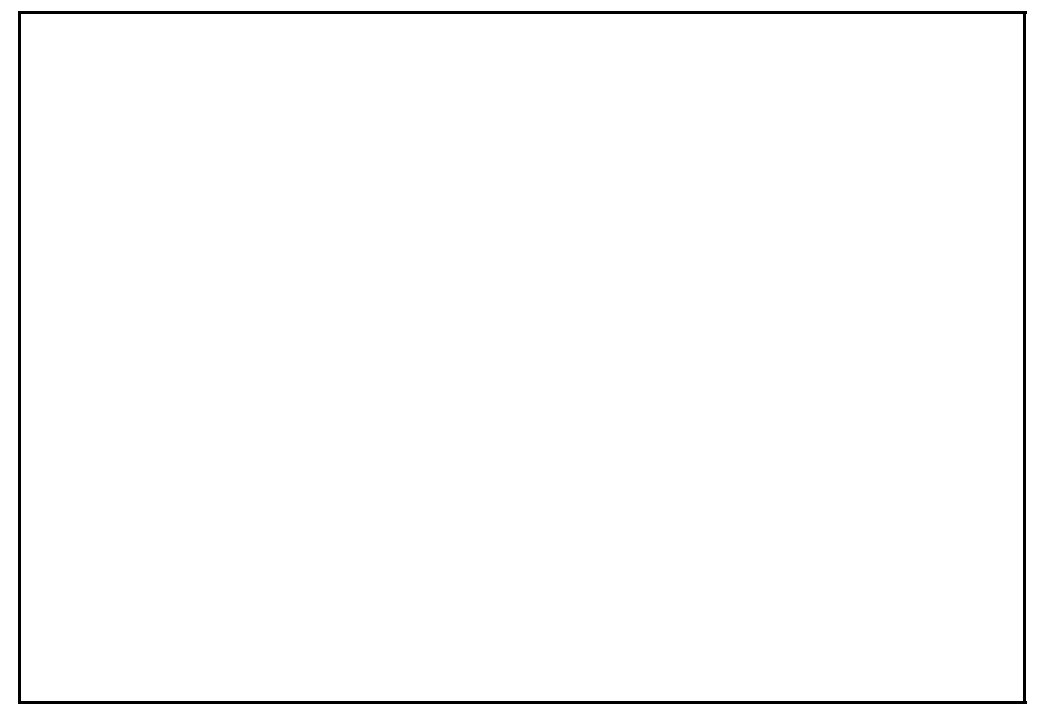

Figure 8: (a) Top left: grey-level image of a section for a fingerprint. (b) Top right: Ridge regions derived from Laplacian of Gaussian $\nabla^{2} G$ of image; $\sigma=7.0$ pixels. (c) Bottom left: Hough transform space derived from end-points of skeleton of ridge regions. (d) Bottom right: Crease line superimposed on the skeleton of ridge regions.

Figure 9: Sequence of structuring elements $L^{1}$ through $L^{8}$ used in the thinning operation.

Figure 10: Structuring elements $E^{1}$ through $E^{8}$ used to identify end-points. 
maxima in the Hough accumulator; see, for example, figures 11 (c) and 12 (c).

\subsection{Detection of local maxima in the Hough accumulator}

Unfortunately, ridge end-points are almost never co-linear, even in the most ideal circumstances such as in the synthetic test patterns which have been used to test the technique (see section 5.1). In the case of real fingerprint patterns, this co-linearity is, at best, approximate (see figures 3 through 8 ). Consequently, the solution-set curves in $r-\theta$ space do not intersect in a single point and the effective local maxima cannot be detected simply by comparing the value of a single accumulator element with a given threshold. Before this comparison can be effected, it is essential to process the accumulator, i.e. the Hough transform space, so that accumulator values in a local region are collected and assigned to a single specific accumulator cell. This is accomplished in the research described in this paper by iteratively re-assigning the value of each accumulator element to one of its neighbours, given that this element is not already the local maximum in the $3 \times 3$ pixel neighbourhood centred on that element. The neighbour to which the value is assigned is required to be the local maximum in that $3 \times 3$ region. This iterative process is continued until no more reassignment can be effected at which point the accumulator comprises a set of isolated points, each of which represents a local maximum.

\subsection{Selection of threshold for isolation of candidate crease lines}

Not all of these local maxima correspond to valid lines in the original image and it is necessary to identify a threshold value which accumulator elements must exceed in order to be considered as candidate crease lines. Since the content of the fingerprint images varies considerably, and hence so too does the resultant form of the Hough accumulator, it is desirable to have this threshold chosen adaptively. This can be accomlished by computing some simple statistics on the distribution of the values in the post-processed Hough accumulator and by basing the threshold on these statistics. In this implementation, the threshold, $T$, is given by:

$$
T=\mu+3 \sigma
$$

where $\mu$ and $\sigma$ are the mean and standard deviation of the values in the postprocessed accumulator, respectively. This threshold is low enough to ensure all crease lines are included but high enough to remove the majority of the accumulator elements.

\subsection{Constraints for the isolation of crease lines}

After application of the threshold on the Hough accumulator, a set of candidate crease lines exists. Not all of these lines do, in fact, join the end-points of several 
distinct ridges.

For example, it is common that a single ridge can be fragmented into a number of sections. Each of these sections give rise to an equal number of roughly co-linear skeletal line segments with two end-points. Since these endpoints are co-linear, they too will give rise to valid local maxima in the Hough accumulator which cannot be identified simply by analysis in the Hough space. However, they can be removed from the set of candidate crease lines by analysing the structure of the original segmented and thinned images.

Other pathological cases also exist. It is possible that lines are formed from several end-points of ridges which are not adjacent in the original image and which do not exhibit the spatial relationship required of ridges forming a secondary crease. Again, since all spatial information is lost in the Hough transform, these lines cannot be detected by analysis of the Hough accumulator. And again, they can be isolated by analysing the structure of the original segmented and thinned images.

Both of these cases can be effectively dealt with by the imposition of two constraints on the lines formed by the ridge end-points.

The first constraint is that the orientation of a crease line should be significantly different to the average orientation of the ridges in the region surrounding the crease line. Specifically, candidate crease lines are removed from consideration if they satisfy the following inequality:

$$
\theta_{r}-\delta_{r}^{1} \leq \theta_{c} \leq \theta_{r}+\delta_{r}^{1}
$$

where $\theta_{r}$ is the average ridge orientation, $\delta_{r}^{1}$ is a tolerance, defined to be $20^{\circ}$ in the implementation described in this paper, and $\theta_{c}$ is the orientation of the crease line. The average ridge orientation is computed as the mean orientation of all adjacent skeleton points in the region defined by two lines, one either side of the crease line, which are parallel to the crease line and equidistant from the crease line by a distance equal to twice the calibrated ridge width.

The second constraint concerns the intersection of ridges by the crease line. In a similar vein to the first constraint, candidate crease lines are removed from consideration if they satisfy the following inequality:

$$
\frac{n_{r}}{l_{c}} \geq \delta_{r}^{2}
$$

where $n_{r}$ is the number of ridge pixels lying on the crease line, $l_{c}$ is the length of the crease line, and $\delta_{r}^{2}$ is the tolerance. $\delta_{r}^{2}$ is defined to be 0.1 for all examples in the implementation described in this paper, unless otherwise stated.

Examples of isolated secondary crease lines are shown in figures 3 (d) through $8(\mathrm{~d})$ and in figures $11(\mathrm{~d})$ and $12(\mathrm{~d})$. 


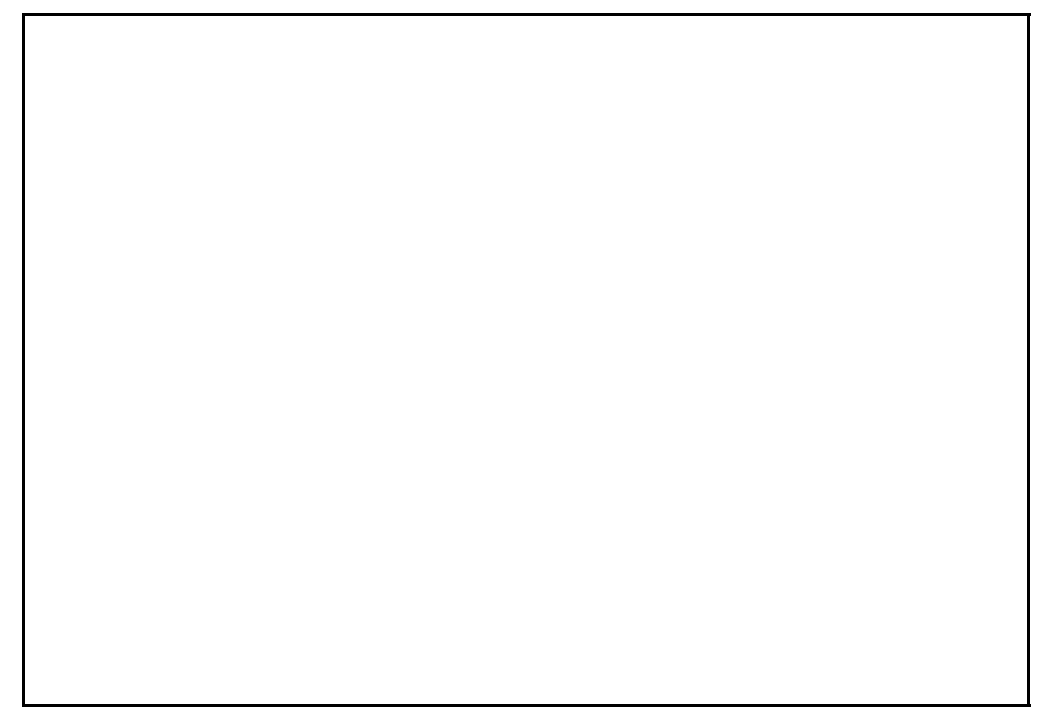

Figure 11: (a) Top left: grey-level image of a synthetic test pattern. (b) Top right: Ridge regions derived from Laplacian of Gaussian $\nabla^{2} G$ of image; $\sigma=7.0$ pixels. (c) Bottom left: Hough transform space derived from end-points of skeleton of ridge regions. (d) Bottom right: Crease line superimposed on the skeleton of ridge regions.

\section{$5 \quad$ Verification of Results}

In order to validate the technique, two forms of test have been run. The first uses synthetic test patterns which have been constructed to assess, in a quantitative manner, how the technique performs as the data degrades in a well-understood manner. The second form of test deals with actual fingerprint data.

\subsection{Tests on synthetic data}

A series of test patterns, each comprising ten ridges, were devised and printed on plain white paper using a laser printer (see, for example, figures 11 and 12). These patterns were printed at an actual size of $8 \mathrm{~mm} \times 6 \mathrm{~mm}$ to ensure that the tests on synthetic data operated at the same magnification and field of view as those on the actual fingerprint data. Consequently, there is a natural variation (or noise) in the image of the test pattern due to the limited resolution of the laser printer, the fibrous texture of the paper, as well as imaging noise. This helps ensure that the tests which are carried out on the synthetic data are as realistic, and representative, as possible.

There are 26 test patterns in total. In the first, each of the ridges has a gap of the same distance as the inter-ridge interval (i.e. the normal distance 


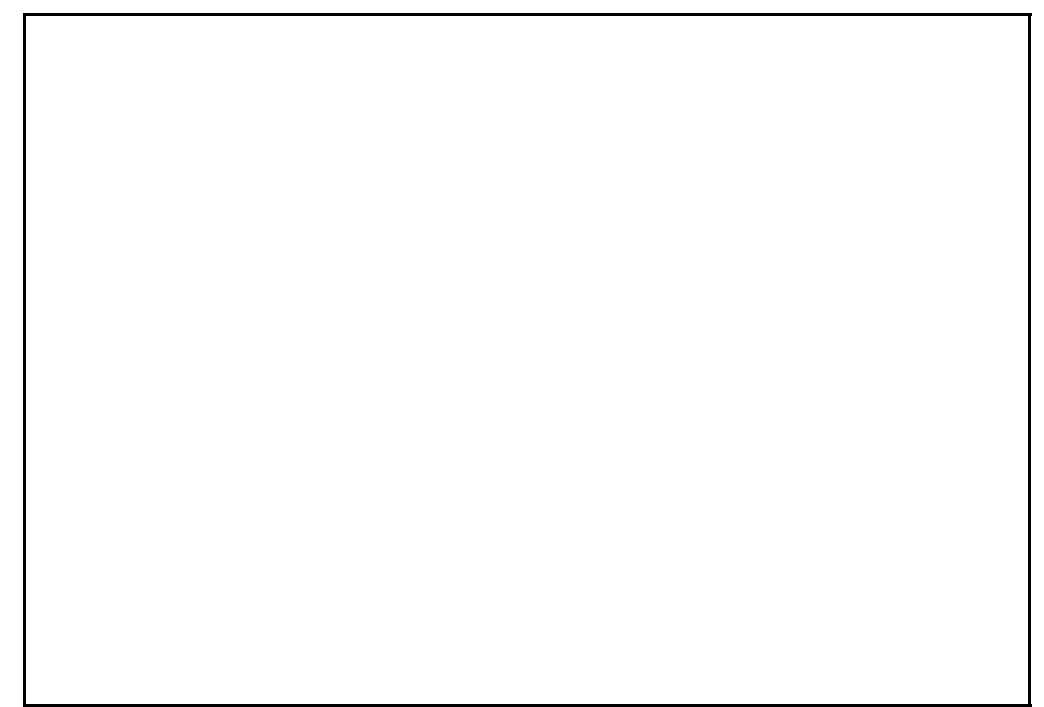

Figure 12: (a) Top left: grey-level image of a synthetic test pattern. (b) Top right: Ridge regions derived from Laplacian of Gaussian $\nabla^{2} G$ of image; $\sigma=7.0$ pixels. (c) Bottom left: Hough transform space derived from end-points of skeleton of ridge regions. (d) Bottom right: Crease line superimposed on the skeleton of ridge regions.

between the ridges) and this gap is at exactly the same position in each ridge: the ridge end-points are all co-linear. The remaining 25 patterns are organized in groups of five, with groups 1, 2, 3, 4, and 5 having $10 \%, 20 \%, 30 \%, 40 \%$, and $50 \%$ of the ridges displaced from the original position, respectively. Within each group, this displacement is varied, with patterns $1,2,3,4$, and 5 having the ridge(s) displaced by $50 \%, 100 \%, 150 \%, 200 \%$, and $250 \%$ of the ridge gap distance.

The technique described in this paper was applied ten times to each pattern and the number of correct crease detections were recorded. Table 1 summarizes the results of this series of tests and details, for each pattern, the rate of correct isolation of the crease. No incorrect crease detections were recorded. These results demonstrate that the technique is consistent and robust. It fails when $40 \%$ or more of the ridges are displaced by $150 \%$ or more of the ridge gap. It should be noted that this failure is due, in every case, to the constraint that the detected ridge line cannot contain more than $10 \%$ of a ridge points along its length (this is the tolerance specified by $\delta_{r}^{2}$ ) and it has been verified that the ridge line would have been detected if this tolerance was altered. 


\begin{tabular}{|c|cccccc|}
\hline $\begin{array}{c}\text { Number of } \\
\text { Displaced Ridges }\end{array}$ & \multicolumn{6}{|c|}{ Displacement of Ridge (\% of ridge gap) } \\
\cline { 2 - 7 } (\% of total) & 0 & 50 & 100 & 150 & 200 & 250 \\
\hline 0 & 100 & - & - & - & - & - \\
10 & - & 100 & 100 & 100 & 100 & 100 \\
20 & - & 100 & 100 & 100 & 100 & 100 \\
30 & - & 100 & 100 & 100 & 100 & 100 \\
40 & - & 100 & 100 & 0 & 0 & 0 \\
50 & - & 100 & 100 & 0 & 0 & 0 \\
\hline
\end{tabular}

Table 1: Results of application of technique on synthetic test patterns: rate of correct isolation of crease(all figures are expressed as percentiles).

\subsection{Tests on real data}

The technique descibed in this paper has also been extensively tested on a somewhat limited data set of fingerprints (in excess of 160 fingerprint regions) and it has proved to be reliable and robust in isolating secondary creases. Representative examples of the results which have been achieved are shown in figures 3 through 8 .

\section{Discussion}

The technique described in this paper works well. Nonetheless, a number of issues should be noted.

First, it has been necessary to use a high imaging magnification, with an attendantly small field of view, in all of the work described. The primary reason for this is to ensure that the features of interest, i.e. the fingerprint ridges, are not under-sampled and that they are well-represented by the digital images which are the object of the analysis. The current field of view is approximately $8 \mathrm{~mm} \times 6 \mathrm{~mm}$ with an image resolution of $256 \times 256$ pixels (and an effective resolution of 32 pixels per millimetre ${ }^{1}$ ). This means that it would require $4 \times 4=16$ images to scan, and analyse, a single fingerprintof dimensions no greater than $32 \mathrm{~mm} \times 24 \mathrm{~mm}$. There is, however, a second reason why so small a field of view is employed. It has been assumed in all of the foregoing that secondary creases are locally linear and that they extend across the greater part of the field of view. If a larger field of view were to be used, these assumptions would no longer be valid.

\footnotetext{
${ }^{1}$ For the sake of comparison, note that the image resolution of finger print database at the National Institute of Standards and Technology in the U.S.A. is 19.7 pixels per millimetre
} 
Second, the spatial filtering of the image in order to emphasise the morphology of the whorls is a key step in the process. The parameter which governs this spatial scale is $\sigma$, the standard deviation in the Laplacian of Gaussian operator. In all of the results cited in this paper, $\sigma=7.0$ pixels. As we noted at the beginning of the paper, this value is determined empirically through calibration procedure, based on the normal distance between the ridges. It should also be noted that the technique is not particularly sensitive to the value of this parameter and, for example, a value of $\sigma=9.0$ pixels has been used successfully in many informal tests.

Third, the extracted ridge end-points are almost never exactly co-linear and, consequently, the Hough transform space is not as well-structured as is often suggested it is in the literature. Thresholding an unprocessed Hough accumulator gave rise to very unstable results. It is essential to post-process the Hough accumulator, in the manner described above, prior to the application of a threshold. This threshold should be, and is, adaptively chosen and, importantly, it should be a lower rather than a higher threshold so that candidate lines are not removed from consideration as secondary creases. Invalid lines can then be ignored after subsequent analysis in the original spatial domain of the semented and skeletonized image, rather than in the Hough transform space.

Two parameters govern the constraints which are used to validate the secondary crease lines: $\delta_{r}^{1}$ and $\delta_{r}^{2}$.

$\delta_{r}^{1}$ is a tolerance on the average orientation of the ridges in the vicinity of the crease line. If the crease line is equal to the the average orientation, plus or minus this tolerance, then the line is deemed not to be a crease line and is ignored. In all of the examples shown in this paper, $\delta_{r}^{1}$ defined to be $20^{\circ}$, unless otherwise stated.

$\delta_{r}^{2}$ is a tolerance on the proportion of a crease line which is allowed to cross ridges. If the proportion of a crease line which contains, or crosses, ridge pixels is greater than this tolerance, then the line is deemed not to be a crease line and is ignored. In all of the examples shown in this paper, $\delta_{r}^{2}$ defined to be 0.1

\section{Conclusions}

The technique for the detection of secondary creases in fingerprints which is described in this paper is robust and works well on all of the data on which it has been tested. This is confirmed by its performance on the synthetic patterns described in the previous section. The clinical usefulness of the technique remains to be assessed by exposing it to a larger data-set and by assessing the correlation between the presence of (automatically) detected secondary creases and the incidence of the disease of which secondary creases may be a physical marker. 


\section{References}

[1] J.S. Weszka, "A survey of threshold selection techniques", Computer Graphics and Image Processing, 7, 259-265 (1978).

[2] J. Serra, Image Analysis and Mathematical Morphology, Academic Press, London (1982).

[3] D. Marr, Vision W.H. Freeman and Co., San Francisco (1982).

[4] D. Marr and E. Hildreth, "Theory of Edge Detection", Proceedings of the Royal Society of London, B207, 187-217 (1980).

[5] D. Vernon and G. Sandini, Parallel Computer Vision - The VIS $\vec{a}$ VIS System, Ellis Horwood, London (1992).

[6] D. Vernon, Machine Vision Prentice-Hall International, London (1991).

[7] P.V.C. Hough, "Method and Means for Recognising Complex Patterms" U.S. Patent $3,069,654,(1962)$. 